\title{
Neoadjuvant treatment for HER2-positive breast cancer
}

\author{
Masahiro Takada, Masakazu Toi \\ Department of Breast Surgery, Kyoto University Hospital, Kyoto, Japan \\ Contributions: (I) Conception and design: M Takada; (II) Administrative support: M Takada; (III) Provision of study materials or patients: M Takada; \\ (IV) Collection and assembly of data: M Takada; (V) Data analysis and interpretation: M Takada; (VI) Manuscript writing: All authors; (VII) Final \\ approval of manuscript: All authors. \\ Correspondence to: Masahiro Takada, MD, PhD. Department of Breast Surgery, Kyoto University Hospital, 54 Kawaharacho, Shogoin, Sakyo-ku, \\ Kyoto, 606-8507, Japan. Email: masahiro@kuhp.kyoto-u.ac.jp.
}

\begin{abstract}
Neoadjuvant therapy has become a standard clinical practice to downsize the tumor and increase the breast-conserving rate. The addition of trastuzumab to neoadjuvant chemotherapy roughly doubles the proportion of patients with HER2-positive breast cancer who achieve pathological complete response (pCR). Patients with pCR show better prognosis compared with those with residual disease after neoadjuvant therapy. Targeting the HER2 pathway with trastuzumab and pertuzumab can further increase the pCR rate. Several studies have shown that neoadjuvant chemotherapy with trastuzumab plus pertuzumab is tolerable, increases the pCR rate compared with trastuzumab alone, and results in about $50-70 \%$ pCR rate. One of the most important studies on neoadjuvant therapy is the KATHERINE trial, in which improved prognostic outcome for patients with residual disease after neoadjuvant therapy was observed. In the trial, improved invasive disease-free survival (DFS) was observed with the administration of postoperative trastuzumab emtansine in patients with HER2-positive breast cancer who had residual disease after neoadjuvant therapy. The indication of neoadjuvant therapy in patients with HER2-positive breast cancer may be changed because the opportunity for residual disease-guided approach, demonstrated in the KATHERINE trial, will be lost when patients had first undergone surgery. Translational studies are promising for further patient selection for HER2-targeted therapy and the development of a novel treatment strategy including PI3K-targeted therapy and immune checkpoint inhibitors. Feasibility studies to evaluate the ability of needle-biopsy to predict pCR after neoadjuvant therapy suggested that standardization and refinements in biopsy procedure (i.e., needle size, number of samples, etc.) are essential for the design of clinical trials of omitted surgery for patients with radiologic complete response.
\end{abstract}

Keywords: Breast neoplasms; neoadjuvant therapies; ErbB-2; trastuzumab; pertuzumab

Submitted Mar 20, 2020. Accepted for publication May 25, 2020.

doi: $10.21037 / \mathrm{cco}-20-123$

View this article at: http://dx.doi.org/10.21037/cco-20-123

\section{Introduction}

Neoadjuvant chemotherapy or preoperative systemic therapy was initially introduced for patients with inoperable breast cancer. Several clinical trials have demonstrated that neoadjuvant therapy increases the proportion of patients who are suitable candidates for breast-conserving surgery and shows the same survival benefit compared with adjuvant therapy $(1,2)$. In addition, neoadjuvant therapy provides information about tumor response in vivo. Several trials and meta-analyses showed a strong correlation between pathological response and prognosis after neoadjuvant therapy $(3,4)$. Patients who achieve a pathological complete response (pCR) after neoadjuvant therapy showed significantly better event-free survival than those with residual disease, especially in patients with HER2-positive and triple-negative breast cancer. In our cohort study of patients with HER2-positive breast cancer treated with neoadjuvant chemotherapy plus trastuzumab, failure to achieve pCR was associated with significantly poorer disease- 
free survival (DFS) than those with pCR [hazard ratio (HR) $1.98,95 \%$ confidence interval (CI): 1.22-3.24] (5).

In the CTNeoBC pooled analysis, there was a slight association between the magnitude of improvement of pCR and the incremental benefit of event-free survival or overall survival (3). In the HER2-positive subgroup of the pooled analysis, only three trials (NOAH trial, TECHNO trial, and GeparQuattro trial) involved trastuzumab administration, and $55 \%$ of the patients in the analysis did not receive trastuzumab. In the NOAH trial, in which patients were randomly assigned to neoadjuvant chemotherapy with or without trastuzumab, the addition of trastuzumab increased pCR rate from $19 \%$ to $38 \%$. This was associated with significant improvement in event-free survival (HR 0.64, 95\% CI: 0.44-0.93) and borderline improvement in overall survival (HR 0.66, 95\% CI: 0.43-1.01) (6,7). The interaction between trastuzumab administration and pCR on event-free survival was statistically significant.

\section{Increasing the proportion of patients who achieve PCR with dual HER2 blockade}

Because of the association between pCR after neoadjuvant therapy and the improved survival outcomes, several trials have been conducted to investigate new treatment regimens which can increase the proportion of patients with pCR. The addition of trastuzumab to neoadjuvant chemotherapy roughly doubles the pCR rate compared to chemotherapy alone (3). The NeoALTTO trial investigated the coadministration of trastuzumab and lapatinib in patients with HER2-positive breast cancer (8). In the NeoALTTO trial, dual inhibition showed higher pCR (ypT0/is+ypN0) rate compared with trastuzumab alone $(46.8 \%$ vs. $27.6 \%$, $\mathrm{P}=0.0007)$. In other dual inhibition trials with trastuzumab and lapatinib, increased pCR rate was observed compared with trastuzumab alone, but there was no statistically significant difference $(9,10)$. In the ALTTO trial, the coadministration of lapatinib and trastuzumab in adjuvant setting did not improve DFS compared with trastuzumab alone (11).

Targeting the HER2 pathway with trastuzumab and pertuzumab can further increase the pCR rate (Table 1). In the NeoSphere trial, patients were randomly assigned to four treatment regimens: (I) trastuzumab and docetaxel (control arm); (II) pertuzumab, trastuzumab, and docetaxel; (III) pertuzumab and trastuzumab; and (IV) pertuzumab and docetaxel (12). Group B showed significantly higher pCR (ypT0/is) rate than group A (45.8\% vs. $29.0 \%, \mathrm{P}=0.014)$.
The number of serious adverse events was similar in groups $\mathrm{A}$ and B. In all the groups, patients with hormone receptornegative disease showed higher pCR rate than those with hormone receptor-positive disease. Interestingly, pCR rate was $16.8 \%$ in group $\mathrm{C}$ in which chemotherapy was not administered. After a 5-year follow-up, an exploratory analysis showed better progression-free survival and DFS for group B than group A; however, the analysis did not have enough power to show the statistical significance (13).

The TRYPHAEA trial investigated the tolerability and activity of pertuzumab and trastuzumab in combination with anthracycline- or carboplatin-based neoadjuvant chemotherapy (14). The trial confirmed a high rate of pCR (ypT0+ypN0) with the dual-HER2 blockade in combination with any chemotherapy regimen (45.3-51.9\%) and showed a low rate of symptomatic left ventricular systolic dysfunction for the anthracycline-based chemotherapy.

The BERENICE trial investigated cardiac safety of pertuzumab and trastuzumab in combination with anthracycline-based chemotherapy regimens (15). This study showed cardiac safety in both dose-dense and standard regimens. The proportions of patients who achieved pCR (ypT0/is+ypN0) were $61.8 \%$ and $60.7 \%$ for dose-dense and standard regimens, respectively.

In HER2-positive subgroup of the GeparSepto trial, patients received paclitaxel or nab-paclitaxel followed by anthracycline-based regimen with concurrent administration of pertuzumab and trastuzumab (16). The pCR rate, defined as ypT0+ypN0, were $53.8 \%$ and $61.8 \%$ for paclitaxel arm and nab-paclitaxel arm, respectively.

The TRAIN-2 trial investigated whether the addition of anthracyclines would improve pCR compared with a carboplatin-taxane regimen (17). In this trial, patients were randomly assigned to paclitaxel and carboplatin with trastuzumab and pertuzumab for nine cycles or anthracycline-based regimen for three cycles, followed by paclitaxel and carboplatin with trastuzumab and pertuzumab for six cycles. The proportion of patients with pCR (ypT0/ is+ypN0) were $68 \%$ for non-anthracycline group and $67 \%$ for anthracycline group $(\mathrm{P}=0.95)$.

The KRISTINE trial compared the efficacy of trastuzumab emtansine (T-DM1) plus pertuzumab, which did not contain systemic chemotherapy regimen, with docetaxel, carboplatin, and trastuzumab plus pertuzumab (18). The proportion of patients with pCR (ypT0/is+ypN0) did not increase with T-DM1 plus pertuzumab, compared with docetaxel, carboplatin, and trastuzumab plus pertuzumab (44\% vs. $56 \%$, respectively). Traditional neoadjuvant 
Table 1 Neoadjuvant trials involving pertuzumab

\begin{tabular}{|c|c|c|c|c|c|}
\hline Trial & Phase & Regimens & $\mathrm{N}$ & pCR rate $(\%)$ & Definition of pCR \\
\hline \multirow{3}{*}{ NeoSphere } & & $\mathrm{DTX}+\mathrm{HP}$ & 107 & 45.8 & \\
\hline & & $\mathrm{HP}$ & 107 & 16.8 & \\
\hline & & $\mathrm{DTX}+\mathrm{P}$ & 96 & 24 & \\
\hline \multirow{2}{*}{ TRYPHEANA } & & $\mathrm{FEC} \rightarrow \mathrm{DTX}+\mathrm{HP}$ & 75 & 45.3 & \\
\hline & & TCbHP & 77 & 51.9 & \\
\hline \multirow[t]{2}{*}{ BERENICE } & rll & $\mathrm{ddAC} \rightarrow \mathrm{PTX}+\mathrm{HP}$ & 199 & 61.8 & ypTO/is+ypNO \\
\hline & & $\mathrm{FEC} \rightarrow \mathrm{DTX}+\mathrm{HP}$ & 201 & 60.7 & \\
\hline \multirow[t]{2}{*}{ TRAIN-2 } & III & TCbHP x9 & 206 & 68 & ypTO/is+ypNo \\
\hline & & FEC $x 3 \rightarrow$ TCbHP $\times 6$ & 212 & 67 & \\
\hline \multirow[t]{2}{*}{ KRISTINE } & III & TCbHP & 221 & 56 & ypTO/is+ypNO \\
\hline & & T-DM1+P & 223 & 44 & \\
\hline \multirow[t]{4}{*}{ JBCRG-20 } & rll & $\mathrm{TCbHP}$ & 51 & 56.9 & ypTO/is+ypNO \\
\hline & & $\mathrm{TCbHP} \rightarrow \mathrm{T}-\mathrm{DM} 1+\mathrm{P}$ & 52 & 71.2 & \\
\hline & & T-DM1+P (responder) & 80 & 62.5 & \\
\hline & & T-DM1+P $\rightarrow$ FEC (non-responder) & 21 & 38.1 & \\
\hline
\end{tabular}

rII, randomized phase II; III, phase III; DTX, docetaxel; H, trastuzumab; P, pertuzumab; FEC, 5-FU + epirubicin + cyclophosphamide; TCbHP, docetaxel + carboplatin + trastuzumab + pertuzumab; PTX, paclitaxel; nab-PTX, nab-paclitaxel; EC, epirubicin + cyclophosphamide; T-DM1, trastuzumab emtansine.

systemic chemotherapy plus trastuzumab and pertuzumab resulted in significantly more patients with pCR than HER2-targeted therapy alone.

In the JBCRG-20 trial, patients were randomly assigned to three treatment regimens: (I) docetaxel, carboplatin, and trastuzumab plus pertuzumab for six cycles; (II) docetaxel, carboplatin, and trastuzumab plus pertuzumab for four cycles followed by T-DM1 plus pertuzumab for four cycles; (III) T-DM1 plus pertuzumab for four cycles, followed by two cycles of same regimen for responders or four cycles of anthracycline-based regimen for non-responders (19). The pCR rates were $57 \%, 71 \%$, and $57 \%$ for group A, group B, and group C, respectively. Group B (69\%) showed significantly higher pCR rate than group A (43\%) and $\mathrm{C}(51 \%)$ in patients with ER-positive cancer, however the pCR rate was not significantly different between the treatment groups in patients with ER-negative cancer
$(67-76 \%)$.

In summary, neoadjuvant chemotherapy with trastuzumab plus pertuzumab is tolerable, increases the pCR rate compared with trastuzumab alone, and results in about $50-70 \%$ pCR rate.

\section{Treatment for patients with pCR after neoadjuvant therapy}

Patients who achieved pCR after neoadjuvant therapy have a significantly better prognosis compared with those with residual disease. The de-escalation of adjuvant treatment may not impair prognosis after surgery for patients with pCR.

There is a debate over if patients with pCR after neoadjuvant therapy with trastuzumab and pertuzumab should only receive adjuvant trastuzumab or receive both 


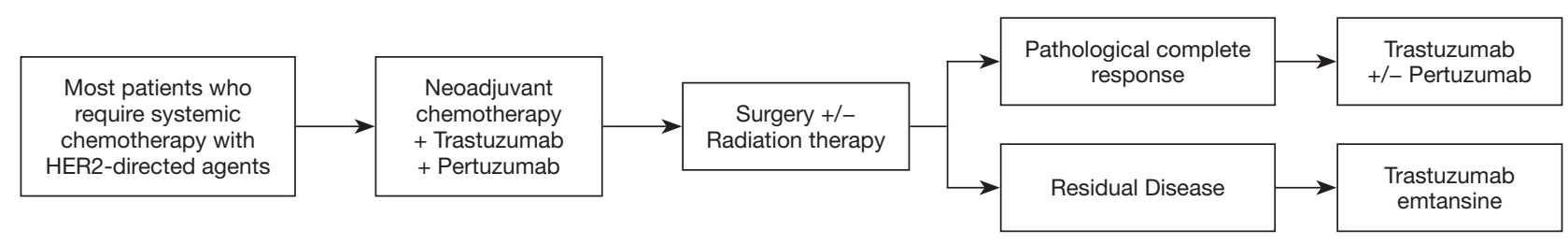

Figure 1 Residual disease-guided approach for HER2-positive breast cancer. Residual disease after neoadjuvant therapy can be used to guide adjuvant therapy to improve prognostic outcome. For most patients who require systemic chemotherapy with HER2-directed agents, neoadjuvant administration of the planned therapy, using the residual disease-guided approach, may provide survival advantages.

trastuzumab and pertuzumab. In a CTNeoBC pooled analysis, although pCR was associated with a favorable event-free survival, a substantial number of patients with pCR experienced disease recurrence after surgery (3). Huober et al. (20) investigated prognostic factors among patients who achieved pCR after neoadjuvant therapy using the data from five clinical trials conducted by German Breast Group, and presented the results at San Antonio Breast Cancer Symposium 2018. They showed that baseline clinical nodal and tumor stage were independently correlated with DFS after surgery in patients with pCR (HR 1.70, 95\% CI: $1.2-2.4$ for nodal stage, and HR 1.61, 95\% CI: $0.97-2.7$ for tumor stage). The results indicate that patients with large tumor size or positive node at disease presentation have a certain risk of recurrence even if they achieve pCR after neoadjuvant therapy. The APHINITY trial, an adjuvant randomized trial, has been able to show that the administration of pertuzumab for 1 year in addition to adjuvant chemotherapy with trastuzumab improved invasive DFS (21). As most of the patients who are recommended for neoadjuvant therapy have node-positive or $\geq \mathrm{T} 2$ tumor, it is acceptable to administer both adjuvant pertuzumab and trastuzumab for patients who underwent neoadjuvant therapy. However, its cost-effectiveness should be carefully evaluated.

\section{Improvement of survival in patients with residual disease after neoadjuvant therapy}

One of the most important studies on neoadjuvant therapy is the Phase 3 KATHERINE trial, which showed improved prognostic outcome for patients with residual disease after neoadjuvant therapy. The randomized trial evaluated the efficacy of adjuvant T-DM1 versus trastuzumab in patients with HER2-positive breast cancer and residual disease after neoadjuvant chemotherapy plus HER2-targeted therapy (22). The primary end point was invasive DFS.
A total of 1,486 patients with residual disease were enrolled. Of those, $18 \%$ were administered trastuzumab plus pertuzumab as neoadjuvant therapy. At the interim analysis, T-DM1 showed significantly better invasive DFS than trastuzumab (HR 0.50, 95\% CI: 0.39-0.64, P<0.001). T-DM1 also reduced distant recurrence compared with trastuzumab (HR 0.60, 95\% CI: 0.45-0.79). A subgroup analysis revealed a consistent benefit of T-DM1 across all the subgroups, even in patients with minimal residual disease after neoadjuvant therapy. Although more adverse events were associated with T-DM1 than with trastuzumab alone, the safety profile was consistent with the previous studies of T-DM1.

About $80 \%$ of participants in the KATHERINE trial received neoadjuvant chemotherapy plus trastuzumab alone. Currently, neoadjuvant chemotherapy with trastuzumab plus pertuzumab is recommended for most patients who are candidates for neoadjuvant therapy. In metastatic HER2positive breast cancer, T-DM1 has clinical activity in patients who have previously received trastuzumab and pertuzumab treatment (23). A subgroup analysis of the KATHERINE trial showed the benefit of T-DM1 in patients who have previously received trastuzumab plus additional HER2directed agent (HR 0.54, 95\% CI: 0.27-1.06).

\section{Indication of neoadjuvant therapy}

Residual disease after neoadjuvant therapy can be used to guide adjuvant therapy to improve prognostic outcome (24). For most patients who require systemic chemotherapy with HER2-directed agents, neoadjuvant administration of the planned therapy by using residual disease-guided approach may provide survival advantages (Figure 1). One of the important clinical advantages of neoadjuvant therapy is the downstaging of tumor which reduces the extent of surgical therapy. Patients with multifocal tumors will often be offered mastectomy even if they receive neoadjuvant 
therapy. However, they will have an option to receive neoadjuvant therapy, through the residual disease-guided approach, to improve the survival outcome. Patients whose tumors are good candidates for breast-conserving surgery at disease presentation ( $\mathrm{T} 1$ or $\mathrm{T} 2$ ) also have an option to receive neoadjuvant therapy due to the same reason.

It is also important not to overtreat patients with lowrisk early stage breast cancer. The adjuvant paclitaxel and trastuzumab (APT) trial is a single-arm adjuvant study involving a weekly administration of paclitaxel plus trastuzumab for 12 weeks followed by 9 months of trastuzumab monotherapy (25). In this trial, patients with HER2-positive tumors measuring up to $3 \mathrm{~cm}$ were enrolled. Of the 406 patients included, over $90 \%$ had tumor less than $2 \mathrm{~cm}$, most patients had node-negative disease, and 64\% had estrogen-receptor positive tumor. The 7-year DFS rate was 93\% (95\% CI: 90-96\%) with four distant recurrences (26). These data show that patients with stage I tumor, especially for estrogen receptor-positive tumor, have excellent longterm outcomes with APT. Therefore, patients with low-risk stage I tumor should first undergo surgery, and consider adjuvant therapy based on the pathological stage.

\section{Translational researches}

Several studies have shown the heterogeneity of HER2positive breast cancer as defined by the ASCO-CAP guideline. In CALBG 40601 and PAMELA trials, patients with HER2-positve tumors were treated with neoadjuvant therapy including trastuzumab and lapatinib, and intrinsic molecular subtype defined by PAM50 was assessed using baseline biopsy specimens $(10,27)$. In these studies, the proportion of patients with HER2-enriched subtype was $31 \%$ and $67 \%$ for CALBG 40601 and PAMELA trials, respectively, and the distribution of the intrinsic subtype was significantly different between hormone receptorpositive and -negative disease. In both studies, the basallike subtype was identified only in patients with hormone receptor-negative disease. Patients with HER2-enriched subtype showed significantly higher pCR rate than nonHER2 enriched subtype (70\% for the CALBG 40601 study and $41 \%$ for the PAMELA study).

A pooled analysis of five trials of trastuzumab and lapatinib showed that PIK3CA mutation is associated with reduced pCR rate in patients with HER2-positive breast cancer (28). In the NeoSphere trial, a non-significant decreased pCR rate was observed consistently across groups in patients with PIK3CA mutation, and especially,
PIK3CA exon 9 mutations were associated with residual disease (29).

Several studies have shown that tumor infiltratinglymphocytes (TILs) are predictive for pCR after neoadjuvant therapy in patients with HER2-positive breast cancer (30-33). In a pooled analysis of neoadjuvant studies, pCR was achieved in 32\% for low TILs group, 39\% for intermediate TILs group, and 48\% for high TILs group (34). In the multivariate analysis, TILs as a continuous marker was predictive for pCR (OR 1.12, 95\% CI: 1.06-1.17, $\mathrm{P}<0.0001)$.

Several studies have shown that circulating tumor cell (CTC) detection is an associated prognosis in both metastatic and primary breast cancer. A meta-analysis showed that the detection of CTC before starting neoadjuvant therapy is associated with overall survival (35). Circulating tumor DNA (ctDNA) analysis has shown promise for the monitoring of treatment response. Two studies showed that the detection of ctDNA after neoadjuvant therapy is associated with the existence of residual disease $(36,37)$. Rothe et al. showed that ctDNA detection before neoadjuvant therapy is associated with decreased pCR rate in a translational study in the NeoALLTO trial (38).

The aforementioned biomarkers are promising for further patient selection for HER2-targeted therapy and in the development of a novel treatment strategy including PI3K-targeted therapy and immune checkpoint inhibitors.

\section{Omission of surgery for patients with radiologic complete response}

In a meta-analysis, neoadjuvant chemotherapy was associated with more frequent local recurrence than adjuvant chemotherapy (39). Increase in local recurrence was evident in the two clinical trials in which many patients did not have breast surgery after neoadjuvant chemotherapy. The meta-analysis indicates that careful tumor localization and adequate local therapy may mitigate local recurrence after neoadjuvant therapy.

As mentioned above, pCR rate after neoadjuvant therapy with trastuzumab and pertuzumab can be up to $70 \%$, and patients with $\mathrm{pCR}$ after neoadjuvant therapy have excellent long-term survival. If we could precisely predict pCR after neoadjuvant therapy, we may omit surgery after neoadjuvant therapy, which would eliminate post-surgical complications and improve quality-of-life. However, current standard imaging modalities cannot accurately predict the status of 
Table 2 Prospective studies on the accuracy of image-guided biopsy to predict pCR after neoadjuvant therapy

\begin{tabular}{lcccc}
\hline Study & N & Eligibility & Type of biopsy & False negative rate (\%) \\
\hline Pooled study (MDACC, SNUH, Royal Marsden) & 166 & cPR/CR cases & VAB/CNB, image-guided & 18.7 \\
MICRA trial & 167 & cPR/CR cases & CNB, image-guided & 37 \\
RESPONDER trial & 398 & cPR/CR cases & VAB, image-guided & 17.8 \\
NRG/BR005 trial & 98 & cCR/near CR cases & CNB, image-guided & 50 \\
\hline
\end{tabular}

cPR, clinical partial response; cCR, clinical complete response; VAB, vacuum assisted biopsy; CNB, core needle biopsy.

residual disease after neoadjuvant therapy $(40,41)$.

The results of four prospective studies which assessed the accuracy of image-guided breast biopsy to predict residual cancer after neoadjuvant therapy were presented at the San Antonio Breast Cancer Symposium 2019 (Table 2). Unfortunately, false negative rate ranged from $17 \%$ to $50 \%$. These studies suggested that standardization and refinements in biopsy procedure (i.e., needle size, number of samples, etc.) is essential in designing the clinical trials of omitted surgery.

Researchers also have to decide how much false negative rate can be allowed for the prediction of $\mathrm{pCR}$ when designing clinical trials of omitted surgery after neoadjuvant therapy. In case of false negative, the opportunity for residual disease-guided approach which was demonstrated in the KATHERINE trial would be lost. As mentioned above, there may be substantial risk of recurrence for patient with large tumor size or positive-node at disease presentation even if they achieve pCR after neoadjuvant therapy. It is essential to develop a novel approach to accurately predict prognosis after neoadjuvant therapy.

\section{Prediction of prognosis after neoadjuvant therapy in HER2-positive breast cancer}

We developed a mathematical model to predict risk of DFS event and brain metastasis (BM) within 5 years after surgery using a multicenter cohort data of 776 patients with HER2positive breast cancer (42). All of the patients received neoadjuvant chemotherapy plus trastuzumab. The area under the receiver operating characteristics curve values were 0.785 for the model to predict DFS and 0.871 for the model to predict BM. What should be noted was that the models could discriminate the prognosis independent of pathological response.

Although our model could discriminate the prognosis at the patient level accurately, there is room for improvement. The integration of novel biomarkers, such as TILs, CTCs, or ctDNA, may enhance the accuracy of the prediction models.

\section{Conclusions}

Neoadjuvant chemotherapy with trastuzumab plus pertuzumab increased pCR rate compared with trastuzumab alone. Residual disease-guided approach, demonstrated in the KATHERINE trial, can improve the prognostic outcome in patients with residual tumor after neoadjuvant therapy. Although clinical advantage of the neoadjuvant therapy is to reduce the extent of surgery by tumor downstaging, neoadjuvant administration of the therapy should be considered as the standard of care for most patients who require adjuvant systemic chemotherapy with HER2-directed agents. Translational researches are promising for further patient selection for HER2-targeted therapy and for the development of a novel treatment strategy including PI3K-targeted therapy and immune checkpoint inhibitors.

\section{Acknowledgments}

We would like to thank Editage (www.editage.com) for English language editing.

Funding: None.

\section{Footnote}

Provenance and Peer Review: This article was commissioned by the Guest Editors (Yutaka Yamamoto and Takayuki Ueno) for the series "Neoadjuvant/Adjuvant Treatment for Early Breast Cancer" published in Chinese Clinical Oncology. The article was sent for external peer review organized by 
the Guest Editors and the editorial office.

Conflicts of Interest: Both authors have completed the ICMJE uniform disclosure form (available at http://dx.doi. org/10.21037/cco-20-123). The series "Neoadjuvant/ Adjuvant Treatment for Early Breast Cancer" was commissioned by the editorial office without any funding or sponsorship. Dr. Takada reports other from Chugai, other from AstraZeneca, other from Pfizer, other from Eli-lilly, other from Eisai, other from Daiichi Sankyo, other from Kyowa-Kirin, grants from Eisai, grants from Nipponkayaku, other from Takeda, other from Nipponkayaku, outside the submitted work. Dr. Toi reports grants and personal fees from Chugai, grants and personal fees from Takeda, grants and personal fees from Pfizer, grants, personal fees and other from Kyowa-Hakko-Kirin, grants and personal fees from Taiho, grants from JBCRG association, grants and personal fees from Eisai, grants, personal fees and other from Daiichi-Sankyo, grants and personal fees from Astra Zeneca, personal fees from Eli Lilly, personal fees from MSD, personal fees from Genomic Health, personal fees from Novartis, personal fees and other from Konica Minolta, grants from Astellas, other from BMS, grants and personal fees from Shimadzu, personal fees from Yakult, grants and personal fees from Nippon Kayaku, grants from AFI technologies, other from Athenex Oncology, outside the submitted work; and Board of directors; JBCRG association, Organisation for Oncology and Translational Research, Kyoto Breast cancer Research Network.

Ethical Statement: The authors are accountable for all aspects of the work in ensuring that questions related to the accuracy or integrity of any part of the work are appropriately investigated and resolved.

Open Access Statement: This is an Open Access article distributed in accordance with the Creative Commons Attribution-NonCommercial-NoDerivs 4.0 International License (CC BY-NC-ND 4.0), which permits the noncommercial replication and distribution of the article with the strict proviso that no changes or edits are made and the original work is properly cited (including links to both the formal publication through the relevant DOI and the license). See: https://creativecommons.org/licenses/by-nc-nd/4.0/.

\section{References}

1. Fisher B, Bryant J, Wolmark N, et al. Effect of preoperative chemotherapy on the outcome of women with operable breast cancer. J Clin Oncol 1998;16:2672-85.

2. Mauri D, Pavlidis N, Ioannidis JP. Neoadjuvant versus adjuvant systemic treatment in breast cancer: a metaanalysis. J Natl Cancer Inst 2005;97:188-94.

3. Cortazar P, Zhang L, Untch M, et al. Pathological complete response and long-term clinical benefit in breast cancer: the CTNeoBC pooled analysis. Lancet 2014;384:164-72.

4. von Minckwitz G, Untch M, Blohmer JU, et al. Definition and impact of pathologic complete response on prognosis after neoadjuvant chemotherapy in various intrinsic breast cancer subtypes. J Clin Oncol 2012;30:1796-804.

5. Takada M, Ishiguro H, Nagai S, et al. Survival of HER2positive primary breast cancer patients treated by neoadjuvant chemotherapy plus trastuzumab: a multicenter retrospective observational study (JBCRG-C03 study). Breast Cancer Res Treat 2014;145:143-53.

6. Gianni L, Eiermann W, Semiglazov V, et al. Neoadjuvant and adjuvant trastuzumab in patients with HER2-positive locally advanced breast cancer (NOAH): follow-up of a randomised controlled superiority trial with a parallel HER2-negative cohort. Lancet Oncol 2014;15:640-7.

7. Gianni L, Eiermann W, Semiglazov V, et al. Neoadjuvant chemotherapy with trastuzumab followed by adjuvant trastuzumab versus neoadjuvant chemotherapy alone, in patients with HER2-positive locally advanced breast cancer (the NOAH trial): a randomised controlled superiority trial with a parallel HER2-negative cohort. Lancet 2010;375:377-84.

8. Baselga J, Bradbury I, Eidtmann H, et al. Lapatinib with trastuzumab for HER2-positive early breast cancer (NeoALTTO): a randomised, open-label, multicentre, phase 3 trial. Lancet 2012;379:633-40.

9. Robidoux A, Tang G, Rastogi P, et al. Lapatinib as a component of neoadjuvant therapy for HER2-positive operable breast cancer (NSABP protocol B-41): an open-label, randomised phase 3 trial. Lancet Oncol 2013;14:1183-92.

10. Carey LA, Berry DA, Cirrincione CT, et al. Molecular Heterogeneity and Response to Neoadjuvant Human Epidermal Growth Factor Receptor 2 Targeting in CALGB 40601, a Randomized Phase III Trial of Paclitaxel Plus Trastuzumab With or Without Lapatinib. J Clin Oncol 2016;34:542-9.

11. Piccart-Gebhart M, Holmes E, Baselga J, et al. Adjuvant Lapatinib and Trastuzumab for Early Human Epidermal Growth Factor Receptor 2-Positive Breast Cancer: Results 
From the Randomized Phase III Adjuvant Lapatinib and/ or Trastuzumab Treatment Optimization Trial. J Clin Oncol 2016;34:1034-42.

12. Gianni L, Pienkowski T, Im YH, et al. Efficacy and safety of neoadjuvant pertuzumab and trastuzumab in women with locally advanced, inflammatory, or early HER2-positive breast cancer (NeoSphere): a randomised multicentre, open-label, phase 2 trial. Lancet Oncol 2012;13:25-32.

13. Gianni L, Pienkowski T, Im YH, et al. 5-year analysis of neoadjuvant pertuzumab and trastuzumab in patients with locally advanced, inflammatory, or early-stage HER2-positive breast cancer (NeoSphere): a multicentre, open-label, phase 2 randomised trial. Lancet Oncol 2016;17:791-800.

14. Schneeweiss A, Chia S, Hickish T, et al. Pertuzumab plus trastuzumab in combination with standard neoadjuvant anthracycline-containing and anthracycline-free chemotherapy regimens in patients with HER2-positive early breast cancer: a randomized phase II cardiac safety study (TRYPHAENA). Ann Oncol 2013;24:2278-84.

15. Swain SM, Ewer MS, Viale G, et al. Pertuzumab, trastuzumab, and standard anthracycline- and taxane-based chemotherapy for the neoadjuvant treatment of patients with HER2-positive localized breast cancer (BERENICE): a phase II, open-label, multicenter, multinational cardiac safety study. Ann Oncol 2018;29:646-53.

16. Loibl S, Jackisch C, Schneeweiss A, et al. Dual HER2blockade with pertuzumab and trastuzumab in HER2positive early breast cancer: a subanalysis of data from the randomized phase III GeparSepto trial. Ann Oncol 2017;28:497-504.

17. van Ramshorst MS, van der Voort A, van Werkhoven $\mathrm{ED}$, et al. Neoadjuvant chemotherapy with or without anthracyclines in the presence of dual HER2 blockade for HER2-positive breast cancer (TRAIN-2): a multicentre, open-label, randomised, phase 3 trial. Lancet Oncol 2018;19:1630-40.

18. Hurvitz SA, Martin M, Symmans WF, et al. Neoadjuvant trastuzumab, pertuzumab, and chemotherapy versus trastuzumab emtansine plus pertuzumab in patients with HER2-positive breast cancer (KRISTINE): a randomised, open-label, multicentre, phase 3 trial. Lancet Oncol 2018;19:115-26.

19. Masuda N, Ohtani S, Takano T, et al. A randomized, 3 -arm, neoadjuvant, phase 2 study comparing docetaxel + carboplatin + trastuzumab + pertuzumab (TCbHP), TCbHP followed by trastuzumab emtansine and pertuzumab (T-DM1+P), and T-DM1+P in HER2positive primary breast cancer. Breast Cancer Res Treat 2020;180:135-46.

20. Huober J, Schneeweiss A, Blohmer JU, et al. Abstract P2-0801: Factors predicting relapse in early breast cancer patients with a pathological complete response after neoadjuvant therapy - Results of a pooled analysis based on the GBG meta-database. Cancer Res 2019;79:Suppl. P2-08-01.

21. von Minckwitz G, Procter M, de Azambuja E, et al. Adjuvant Pertuzumab and Trastuzumab in Early HER2Positive Breast Cancer. N Engl J Med 2017;377:122-31.

22. von Minckwitz G, Huang CS, Mano MS, et al. Trastuzumab Emtansine for Residual Invasive HER2Positive Breast Cancer. N Engl J Med 2019;380:617-28.

23. Dzimitrowicz H, Berger M, Vargo C, et al. T-DM1 Activity in Metastatic Human Epidermal Growth Factor Receptor 2-Positive Breast Cancers That Received Prior Therapy With Trastuzumab and Pertuzumab. J Clin Oncol 2016;34:3511-7.

24. Pusztai L, Foldi J, Dhawan A, et al. Changing frameworks in treatment sequencing of triple-negative and HER2positive, early-stage breast cancers. Lancet Oncol 2019;20:e390-e396.

25. Tolaney SM, Barry WT, Dang CT, et al. Adjuvant paclitaxel and trastuzumab for node-negative, HER2positive breast cancer. N Engl J Med 2015;372:134-41.

26. Tolaney SM, Guo H, Pernas S, et al. Seven-Year FollowUp Analysis of Adjuvant Paclitaxel and Trastuzumab Trial for Node-Negative, Human Epidermal Growth Factor Receptor 2-Positive Breast Cancer. J Clin Oncol 2019;37:1868-75.

27. Llombart-Cussac A, Cortes J, Pare L, et al. HER2enriched subtype as a predictor of pathological complete response following trastuzumab and lapatinib without chemotherapy in early-stage HER2-positive breast cancer (PAMELA): an open-label, single-group, multicentre, phase 2 trial. Lancet Oncol 2017;18:545-54.

28. Loibl S, Majewski I, Guarneri V, et al. PIK3CA mutations are associated with reduced pathological complete response rates in primary HER2-positive breast cancer: pooled analysis of 967 patients from five prospective trials investigating lapatinib and trastuzumab. Ann Oncol 2016;27:1519-25.

29. Bianchini G, Kiermaier A, Bianchi GV, et al. Biomarker analysis of the NeoSphere study: pertuzumab, trastuzumab, and docetaxel versus trastuzumab plus docetaxel, pertuzumab plus trastuzumab, or pertuzumab plus docetaxel for the neoadjuvant treatment of HER2- 
positive breast cancer. Breast Cancer Res 2017;19:16.

30. Loi S, Michiels S, Salgado R, et al. Tumor infiltrating lymphocytes are prognostic in triple negative breast cancer and predictive for trastuzumab benefit in early breast cancer: results from the FinHER trial. Ann Oncol 2014;25:1544-50.

31. Salgado R, Denkert C, Campbell C, et al. TumorInfiltrating Lymphocytes and Associations With Pathological Complete Response and Event-Free Survival in HER2-Positive Early-Stage Breast Cancer Treated With Lapatinib and Trastuzumab: A Secondary Analysis of the NeoALTTO Trial. JAMA Oncol 2015;1:448-54.

32. Ignatiadis M, Van den Eynden G, Roberto S, et al. Tumor-Infiltrating Lymphocytes in Patients Receiving Trastuzumab/Pertuzumab-Based Chemotherapy: A TRYPHAENA Substudy. J Natl Cancer Inst 2019;111:69-77.

33. Denkert C, von Minckwitz G, Brase JC, et al. Tumorinfiltrating lymphocytes and response to neoadjuvant chemotherapy with or without carboplatin in human epidermal growth factor receptor 2-positive and triple-negative primary breast cancers. J Clin Oncol 2015;33:983-91.

34. Denkert C, von Minckwitz G, Darb-Esfahani S, et al. Tumour-infiltrating lymphocytes and prognosis in different subtypes of breast cancer: a pooled analysis of 3771 patients treated with neoadjuvant therapy. Lancet Oncol 2018;19:40-50.

35. Bidard FC, Michiels S, Riethdorf S, et al. Circulating Tumor Cells in Breast Cancer Patients Treated by Neoadjuvant Chemotherapy: A Meta-analysis. J Natl

Cite this article as: Takada $M$, Toi $M$. Neoadjuvant treatment for HER2-positive breast cancer. Chin Clin Oncol 2020;9(3):32. doi: $10.21037 /$ cco-20-123
Cancer Inst 2018;110:560-7.

36. McDonald BR, Contente-Cuomo T, Sammut SJ, et al. Personalized circulating tumor DNA analysis to detect residual disease after neoadjuvant therapy in breast cancer. Sci Transl Med 2019;11:eaax7392.

37. Moss J, Zick A, Grinshpun A, et al. Circulating breastderived DNA allows universal detection and monitoring of localized breast cancer. Ann Oncol 2020;31:395-403.

38. Rothe F, Silva MJ, Venet D, et al. Circulating Tumor DNA in HER2-Amplified Breast Cancer: A Translational Research Substudy of the NeoALTTO Phase III Trial. Clin Cancer Res 2019;25:3581-8.

39. Early Breast Cancer Trialists' Collaborative Group (EBCTCG). Long-term outcomes for neoadjuvant versus adjuvant chemotherapy in early breast cancer: metaanalysis of individual patient data from ten randomised trials. Lancet Oncol 2018;19:27-39.

40. Marinovich ML, Houssami N, Macaskill P, et al. Metaanalysis of magnetic resonance imaging in detecting residual breast cancer after neoadjuvant therapy. J Natl Cancer Inst 2013;105:321-33.

41. Schaefgen B, Mati M, Sinn HP, et al. Can Routine Imaging After Neoadjuvant Chemotherapy in Breast Cancer Predict Pathologic Complete Response? Ann Surg Oncol 2016;23:789-95.

42. Takada M, Sugimoto M, Masuda N, et al. Prediction of postoperative disease-free survival and brain metastasis for HER2-positive breast cancer patients treated with neoadjuvant chemotherapy plus trastuzumab using a machine learning algorithm. Breast Cancer Res Treat 2018;172:611-8. 